\title{
A novel multiple-windows blending of CT images in red-green-blue (RGB) color space: Phantoms study
}

\author{
C. Anam ${ }^{1, A}$, W.S Budi',,A, F. Haryanto3,, , T. Fujibuchi4,C, G. Dougherty5,D \\ A Department of Physics, Faculty of Mathematics and Natural Sciences, \\ Diponegoro University \\ B Department of Physics, Faculty of Mathematics and Natural Sciences, \\ Bandung Institute of Technology \\ C Department of Health Sciences, Faculty of Medical Sciences \\ D Applied Physics and Medical Imaging, California State University Channel Islands \\ ${ }^{1}$ ORCID: 00oo-0003-0156-6797, anam@fisika.undip.ac.id \\ 2 ORCID: oooo-0003-1651-1297, wahyu.sb@fisika.undip.ac.id \\ 3 ORCID: 0000-0002-6353-8460, freddy@fi.itb.ac.id \\ 4 ORCID: 0000-0001-9713-6155, fujibuch@hs.med.kyushu-u.ac.jp \\ 5 ORCID: o0oo-0003-3187-3595, Geoff.Dougherty@csuci.edu
}

\begin{abstract}
This study develops an algorithm for multiple-windows blending of CT images in redgreen-blue (RGB) color space. A CT image is windowed at three different combinations of window width (WW) and window level (WL) values. The first window image is then colorized red (R), the second window image is colorized green (G), and the third window image is colorized blue (B). These three images, representing three combinations of window settings, can then be represented as a single RGB color image. The technique was applied to axial images of TOS-phantom with various objects and anthropomorphic phantom. This multiplewindows blending enables the result of multiple-windows to be presented in a single view without any subsequent changes to the windows settings. This multiple-windows blending is able to visualize many tissues of interest simultaneously with high contrast depending on the initial windows settings. The blending of soft tissue, bone, and lungs windows in thoracic images, for example, produces an image in which the details of soft tissues, bones, and lung nodules can be seen simultaneously in one view. Multiple-windows blending can potentially hasten image interpretation because more information is viewed in a single image. However, this novel method needs the radiologist to adapt to interpreting the new multiple-windows blended image. The method also requires standardization and optimization.
\end{abstract}

Keywords: multiple-windows, window blending, image representation, window width, window level.

\section{Introduction}

CT scans are used extensively in medical applications because they produce excellent quality images [1-3] with very fast data acquisition [4]. One of the advantages of CT images compared to images from other radiological modes is their very high contrast
[5]. CT scan images can distinguish between two objects having a contrast of only about $0.5 \%$ [6], so very small structures inside the body can be investigated with excellent detail.

Axial CT images are two dimensional (2D) mappings of the linear at- 
tenuation of various body tissues expressed in Hounsfield units (HU) [7]. The HU scale has a very wide range, i.e. from -1000 to more than +3000 [8]. For example, the pixel value for air is about $-1000 \mathrm{HU}$, fat is between -50 and $-200 \mathrm{HU}$, the lungs are between 200 and $-500 \mathrm{HU}$, muscles are between +25 and +40 HU [1], and solid bones can be more than $+3000[8,9]$. However the most computer monitors can only show 256 separate grays [8], and the human eye can distinguish narrower range than computer monitor. We generally employ a windowing technique, where we choose a range of HU values of interest to be displayed, specified by a window width (WW) and a window level (WL). Window width is the range of HU values to be displayed, while the window level is the middle value of the range [8]. Thus, with this windowing technique, the contrast of specific tissues of interest in the images is enhanced.

In clinical applications there are several types of windows, such as soft tissue, bone, lung, and liver [10-12]. However, displaying a CT scan image with only one type of window can have limitations. For instance, with a soft tissue window, existing tumors in the soft tissue will appear very clearly. However, if the tumor has infiltrated the bone, then that tumor may not be as well visualized on the soft tissue window. To be able to see tumors in bone, it is necessary to change the type of window into a bone window [13]. Some lesions in the liver do not appear with the soft tissue window setting. Mayosmith et al. [14] added a liver window to an abdominal CT image, which is usually interpreted using a standard (i.e. soft tissue) window. They reported that using an additional liver window produced an increase of $3.1 \%$ in the detection of liver lesions compared to a standard (soft tissue) window. Similar results were reported by
Sabouri et al. [15]. These examples indicate that the use of multiple windows may improve the diagnostic accuracy of a CT scan [14-17]. However, interpretation using multiple windows takes additional time and more effort from the radiologists. It is important to develop multiple-windows blending so that the results of multiple windows can be integrated into a single image (i.e., one view).

Previously, Pizer et al. [18] developed adaptive histogram equalization (AHE) that can display a wide range of CT image using only a limited range. One disadvantage of AHE is that the noise component becomes very dominant $[18,19]$. To address this problem, Pizer et al. [20] have proposed a contrast-limited adaptive histogram equalization (CLAHE) as a variant of AHE [20]. Subsequently, multiscale adaptive histogram equalization (MA$\mathrm{HE}$ ) is proposed for automated simultaneous display of the full dynamic range of a CT image [21, 22]. Recently, Mandell et al. [23] developed a multiple-windows blending technique with their relative attenuation-dependent image overlay (RADIO) algorithm, which can display all $\mathrm{HU}$ value ranges in a single view without changing the WW and WL values. However, all previous techniques changed the standard appearance of tissues and may be confusing to radiologists. For example, radiologists have been trained to expect fat to look darker than soft tissue because of its smaller attenuation [24, 25], but the techniques may produce a lighter image of fat than soft tissue. In the current study, we propose a new technique of window blending by utilizing a color image in the red-greenblue (RGB) plane. The three images are then combined to form a single blended RGB color image. To demonstrate its usefulness, we have applied the method to images of TOS-phantom with various objects, thoracic anthro- 
pomorphic phantom, head of patient, and thorax of patient.

\section{Methods}

\subsection{The windowing pro- cess}

In the windowing technique, the range of CT values displayed is called the window width (WW) and the middle value of the range is called the window level (WL). CT values are converted and displayed as 8-bit pixel values using a linear function [7]:

$$
I_{X}=255\left\{\begin{array}{l}
0 \quad \text { for } \mathrm{CT} \text { values } \leq W L-\frac{W W}{2} \\
W W^{-1}\left(\mathrm{CT} \text { value }-W L+\frac{W W}{2}\right)(1) \\
1 \quad \text { for CT values } \geq W L+\frac{W W}{2}
\end{array}\right.
$$

Only CT values within the WW range are displayed as gray values, increasing linearly through the window. $\mathrm{CT}$ values below the WW range are always zero, while those above WW are always 255 (Fig. 1).

The WW and WL values are typically specific for a particular examination. Several types of windows are shown in Table 1. For instance, to see the contrast of objects in the lungs, a lung window is used with WW of 2000 HU and WL of -200 HU. This corresponds to HU values from $-1,200$ to +800 . In the lung window setting, the contrast in the lung area looks very good, but other objects (i,e. the bone) do not display well. To see bone with good contrast, the window setting should be changed to a bone window with WW of 1500 and WL of +300 .

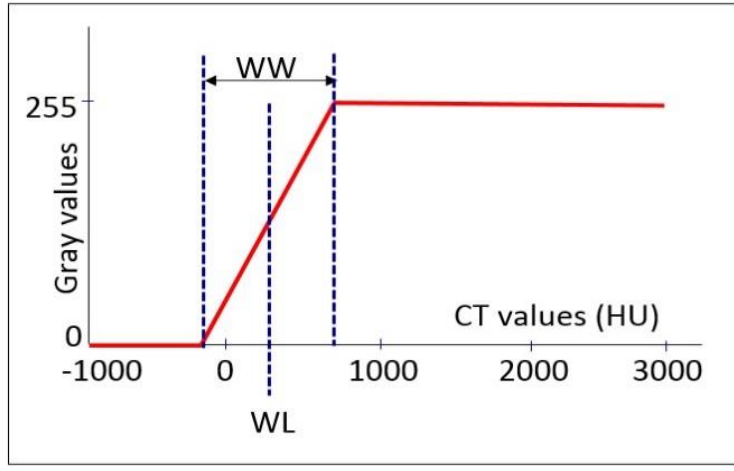

Fig. 1. Graph to illustrate the transformation of CT values to pixel values in the windowing technique, using a linear function. The range of CT values displayed span the window width (WW) and the middle value of the range is the window level (WL).

\subsection{Window blending in RGB space}

Image blending in red-green-blue (RGB) space is shown in Fig. 2. The first windowing represents the red plane, the second windowing representing the green plane, and the third windowing representing the blue plane.

Table 1. Various WW and WL values for different types of window settings used in clinical application.

\begin{tabular}{|l|c|c|}
\hline $\begin{array}{l}\text { Type of } \\
\text { window }\end{array}$ & $\begin{array}{l}\text { Window } \\
\text { width (WW) }\end{array}$ & $\begin{array}{l}\text { Window } \\
\text { level (WL) }\end{array}$ \\
\hline Lung [7] & 2000 & -200 \\
\hline $\begin{array}{l}\text { Soft tissue } \\
{[7]}\end{array}$ & 350 & +50 \\
\hline Bone [7] & 1500 & +300 \\
\hline Liver [16] & 150 & +75 \\
\hline
\end{tabular}

The three results of the windowing are then merged to form a single RGB image. The resulting pixel values after windowing have a range from o to 255 , indicating the intensity of the red, green and blue colors respectively 
(Figure 3). RGB color values can be treated as probabilities in the range $0-1$ if each pixel value is divided by 255 . Since the new image is a blending of the three planes, if the matrix size for one plane is $512 \times 512$, then the matrix size of the color image is $512 \times 512 \times 3$.

$$
I_{R G B}=I(R, G, B)
$$

The resulting image allows each pixel to have a color probability as a combination of three values, each with
256 discrete values between 0 and 1 . If a pixel has a values $(0,0,0)$ then that pixel is black, and if its values are $(1,1,1)$ then the pixel is white. If the pixel has values $(1,0,0)$ the pixel is red, $(0,1,0)$ produces a green pixel, $(0,0,1)$ produces a blue pixel, $(1,1,0)$ produces a yellow pixel , $(1,0,1)$ produces a magenta pixel, and $(0,1,1)$ produces a cyan pixel.

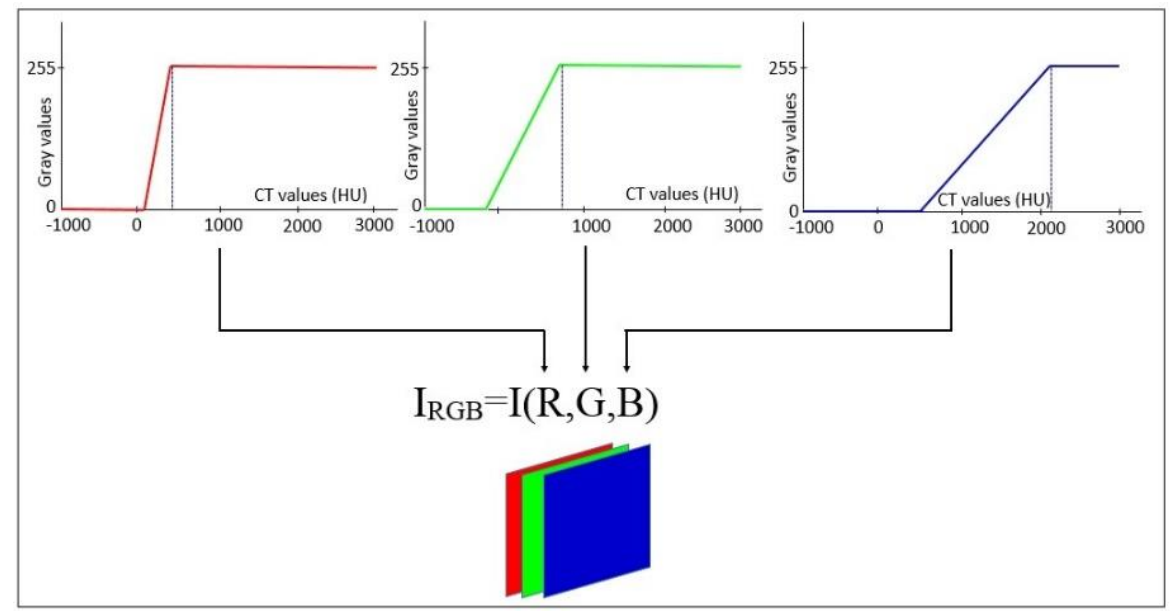

Fig. 2. The result of multiple-windows combined in red-green-blue (RGB) space. The first window results is for the red plane, the second window results is for the green plane, and the third window results is for the blue plane.

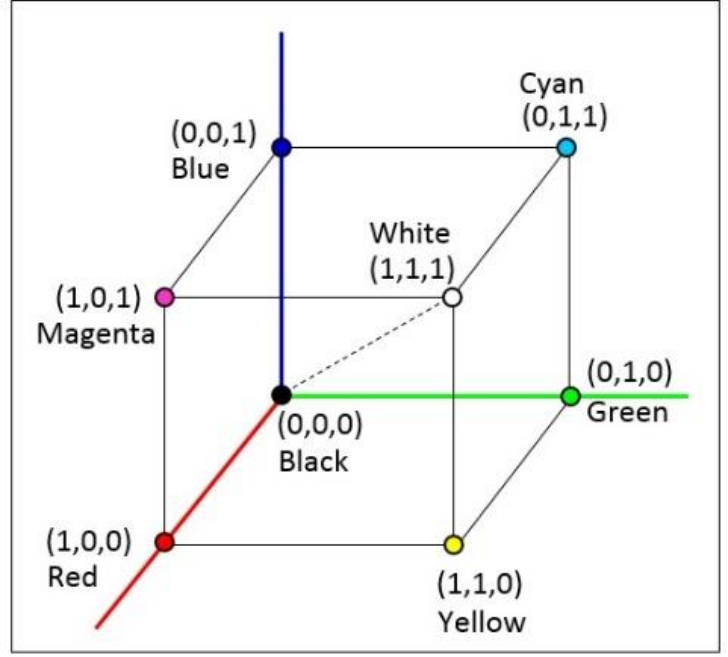

Fig. 3. Schematic diagram of redgreen-blue axes in RGB color space.

\subsection{Phantoms and pa- tients images}

In this study, multiple-windows blending was applied to TOS-phantom (Toshiba Medical Systems, Co., Ltd., Tokyo, Japan) (Fig. 4(a)) and anthropomorphic phantom (Kyoto Kagaku Co., Ltd., Kyoto, Japan) (Fig. 4(b)). The TOS-phantom was used in the module consisted of five cylindrical objects of air, Delrin $\AA$, acrylic, nylon, and polypropylene. The HU values of these five objects were $-990,340$, 125, 100 and $-100 \mathrm{HU}$ for air, Delrin $\AA$, acrylic, nylon, and polypropylene, respectively. The five objects were located inside the water. In the image of the TOS-phantom, we used the win- 
dows settings for liver, soft tissue and lungs. We also applied to the thoracic area of anthropomorphic phantom using the window settings for soft tissue, bone, and lungs as standard windows of chest CT [22]. The multiplewindows blending was also implemented to the head and thoraric images of patients.
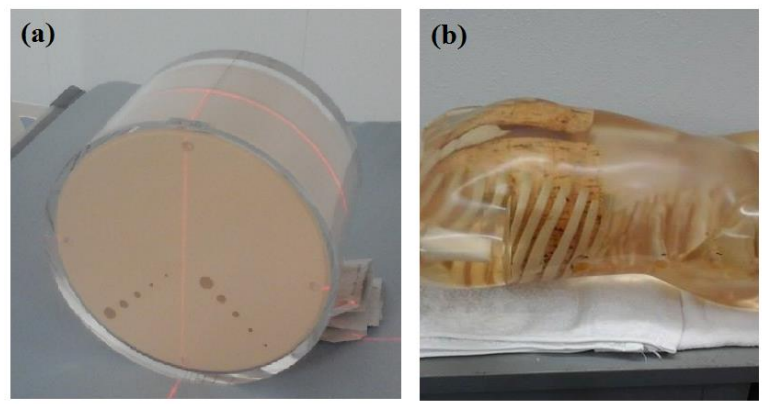

Fig. 4. Phantoms used for multiple windows blending. (a) TOS-phantom, and (b) Anthropomorphic phantom.
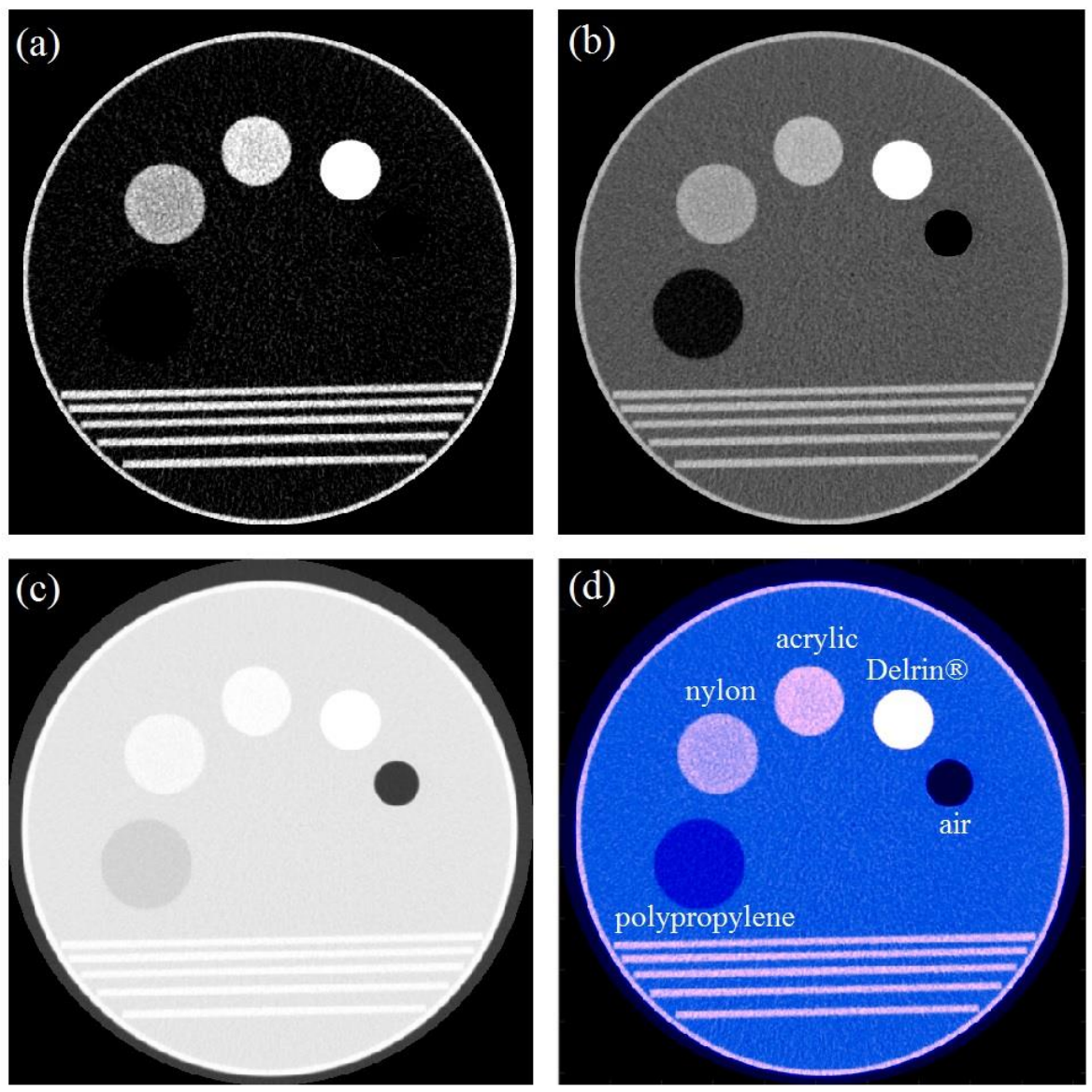

Fig. 5. Images of a TOS-phantom with different window settings. (a) Liver window, (b) Soft-tissue window, (c) Lung window, and (d) Blended of these three windows. 
From these three windows, there are six (3!) combinations of the blended images. The images of six combinations for liver, soft tissue and lung windows in TOS-phantom are shown in Fig. 6. It appears that the image of all combinations can distinguish five objects and backgrounds. It appears
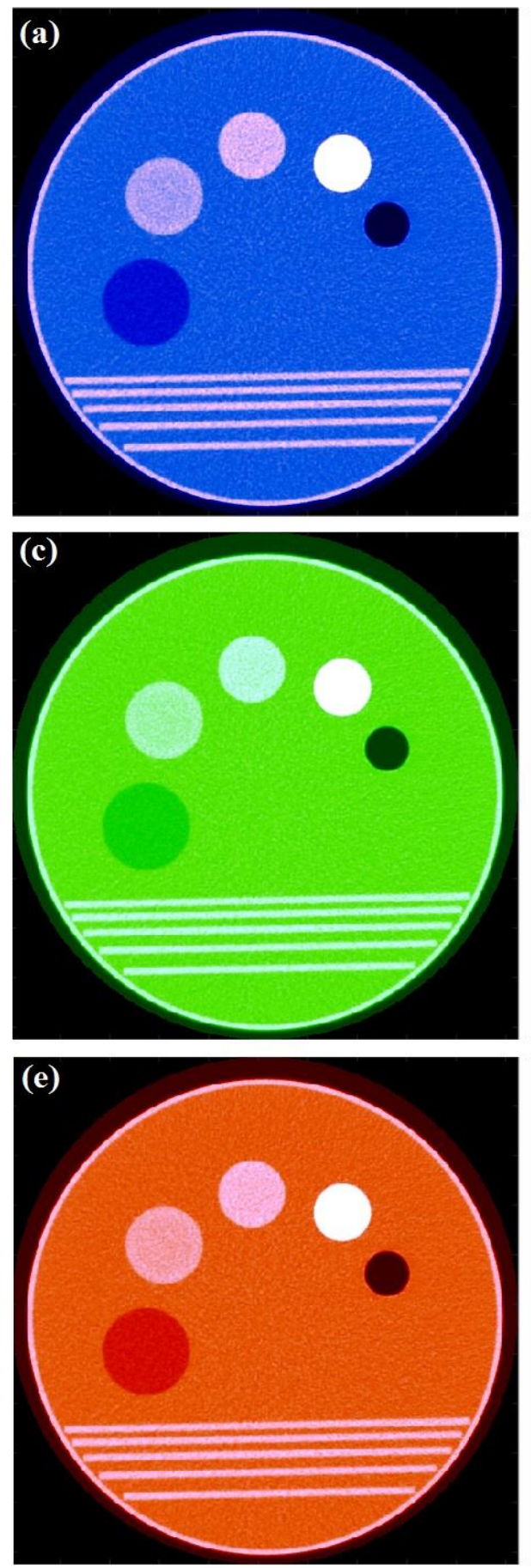

Fig. 6. The images from combination of liver, soft tissue, and lung windows. (a) Liver-soft tissue-lung, (b) Liver-lung-soft tissue, (c) Soft tissue-lung-liver, (d) Soft tissue-liver-lung, (e) Lung-soft tissue-liver, and (f) Lung-liver-soft tissue.

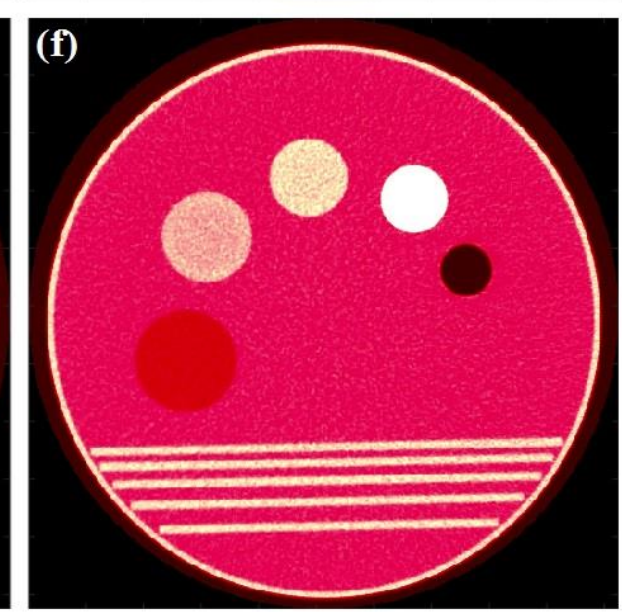

that the most dominant window is the lung window. If the lung window is on blue plane then the background is blue ( $a$ and $d$ ), and if it is on green plane then the background is green (b and c), and the background is red when the lung window is on red plane (e and f).
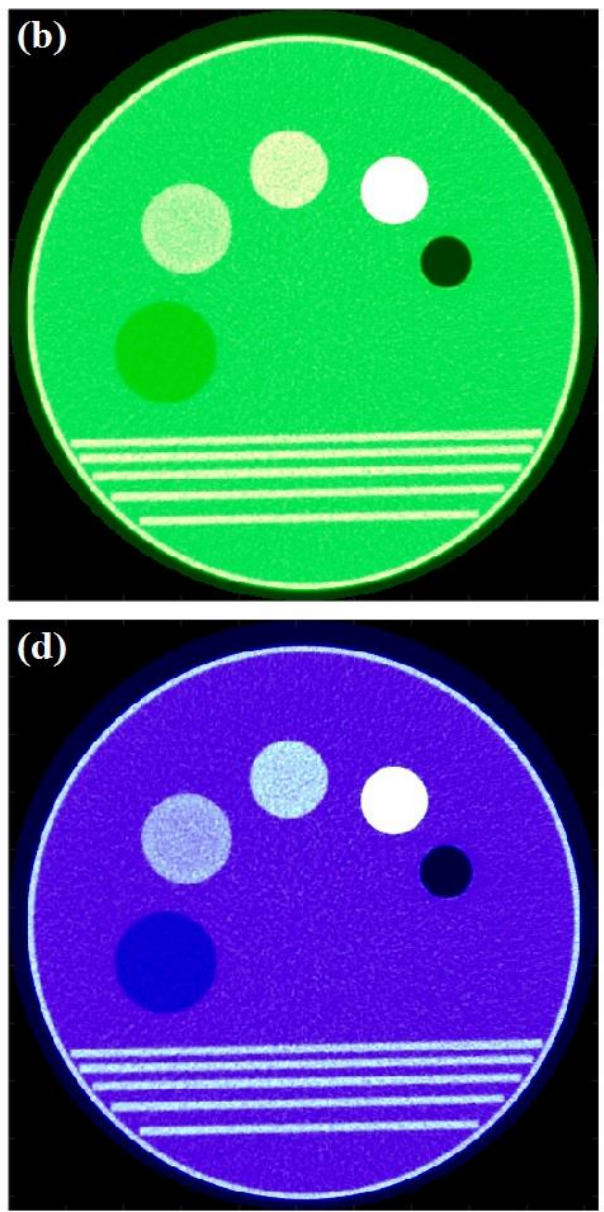

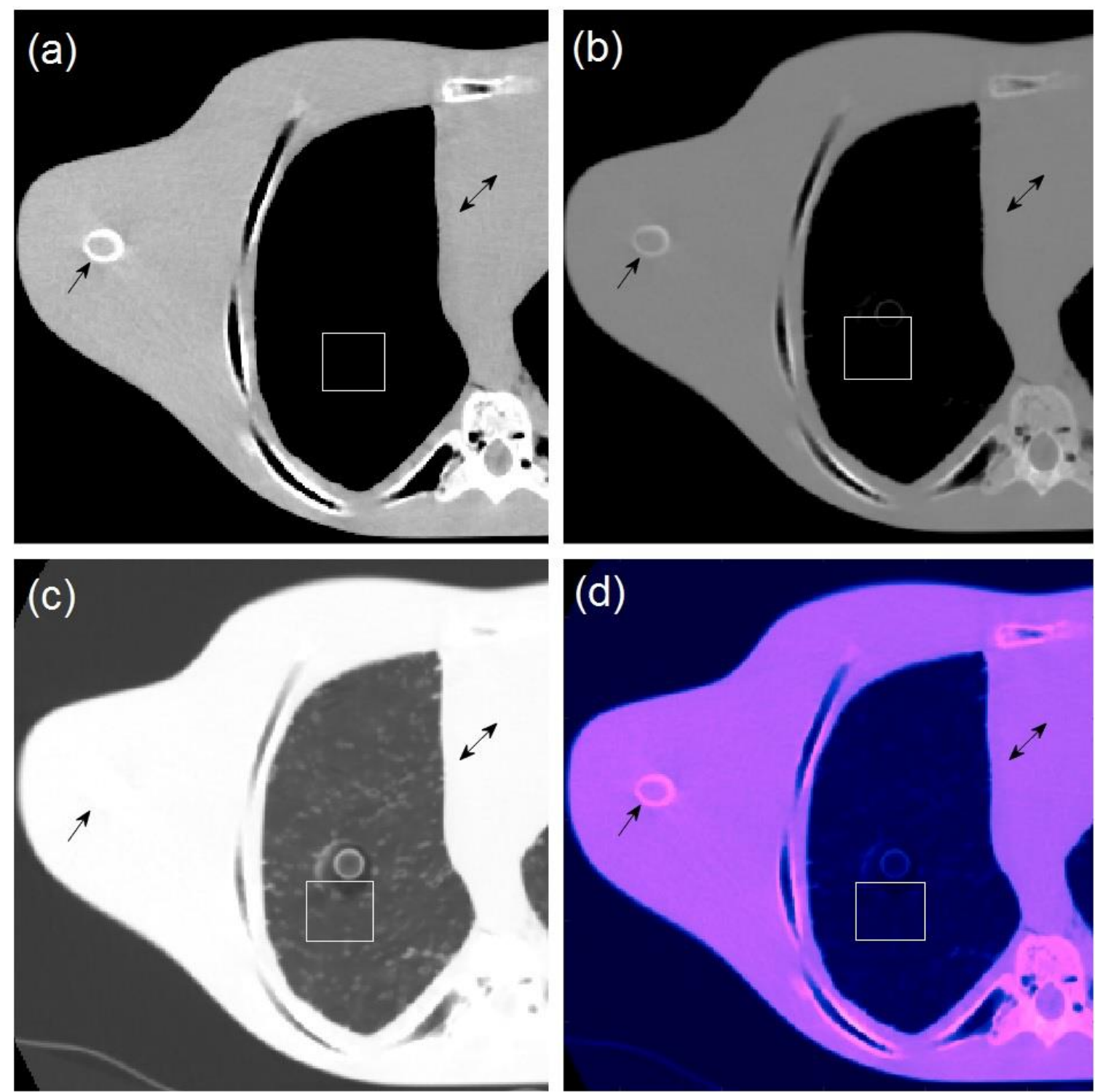

Fig. 7. Images of a thoracic anthropomorphic phantom displayed with different window settings. (a) Soft tissue window, (b) Bone window, (c) Lung window, and (d) Blended of these three windows. A single arrow indicates a humerus bone, a white box indicates nodules in the lung, and a double arrow indicates a soft tissue of the heart.

A typical thoracic image of anthropomorphic phantom displayed with the soft tissue window setting is shown in Fig. $7(\mathrm{a})$, with the bone window setting is shown in Fig. $7(\mathrm{~b})$, and with the lung window setting is shown in Fig. $7(\mathrm{c})$, and the blended of these windows is shown in Fig. $7($ d). Fig. 5(a) shows that the contrast of soft tissues is high using the soft tissue window setting (indicated by double arrow), while the contrast of the lung (indicated by white box) and bone area (indicated by single arrow) are very low. Using the bone window setting, the contrast of the bone is good, but the contrast of the soft tissue and lung areas are poor (Fig. 7(b)). Using the lung window setting, the contrast of the lung is good but the contrast of the soft tissue is very poor (Fig. $7(\mathrm{c})$ ). In the blending of the soft tissue-bone-lung windows, the soft tissues, bones and lung nodules all have high contrast (Fig. $7(\mathrm{~d})$ ). 

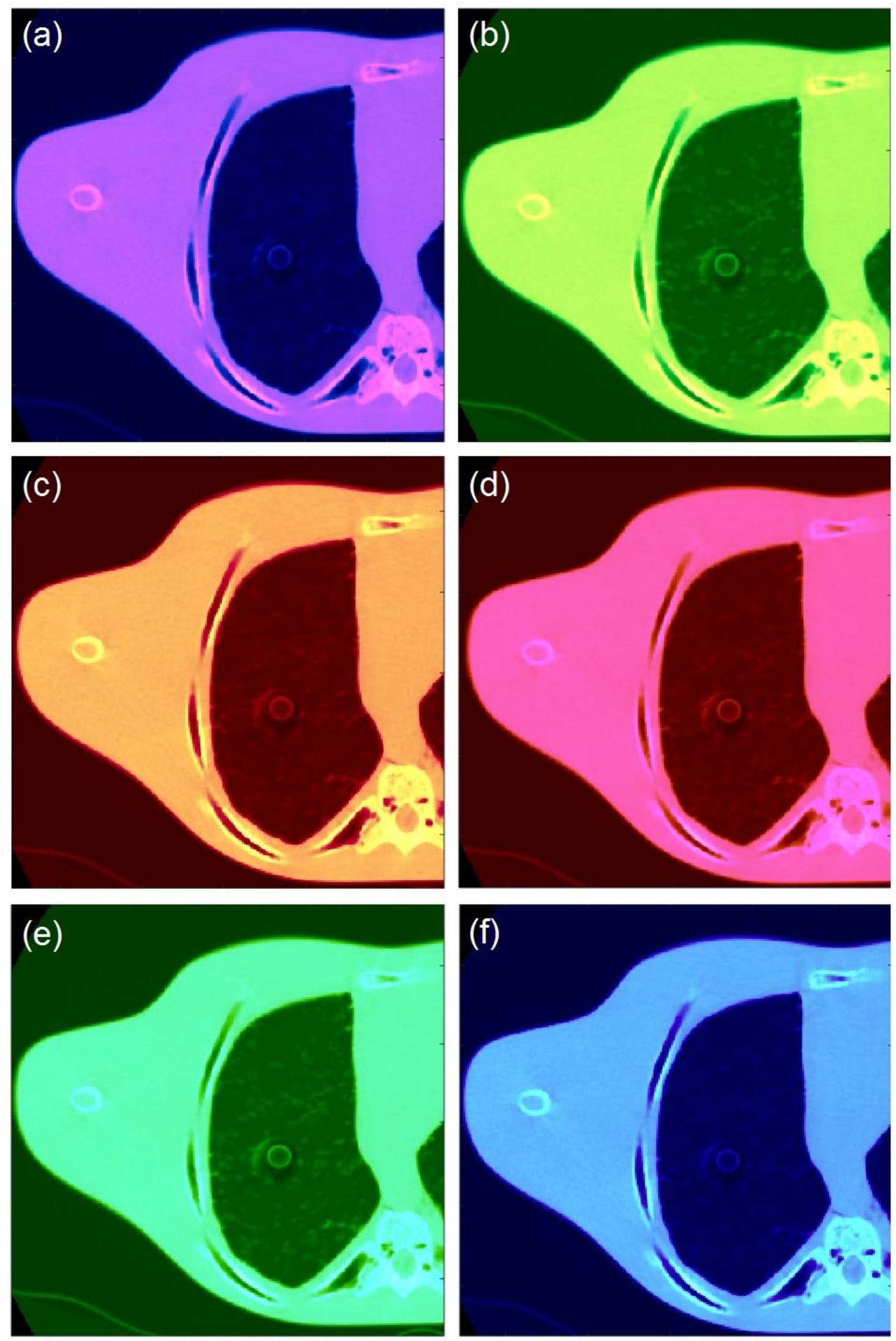

Fig. 8. The images from combination of soft tissue, bone, and lung windows. (a) Soft tissue-bone-lung, (b) Soft tissue-lung-bone, (c) Lung-soft tissue-bone, (d) Lungbone-soft tissue, (e) Bone-lung-soft tissue, (f) Bone-soft tissue-lung. 
In the thoracic anthropomorphic images there are also six combinations. The six combinations for the soft tissue, bone and lung windows are shown in Fig. 8. It shows that the images of all combinations can clearly distinguish soft tissue, humerus bone and lung nodules. However, eye response is different for each of these colors. Fig. 7 shows that the most dominant of view perception is the green plane. Therefore, if the lung window is on the green plane then the lung nodule appears more clearly (b and e), and if the bone window is on the green plane then the humerus bone looks more obvious (a and d). Similarly, if the soft tissue window is on the green plane then soft tissue appears more clearly (c and f).
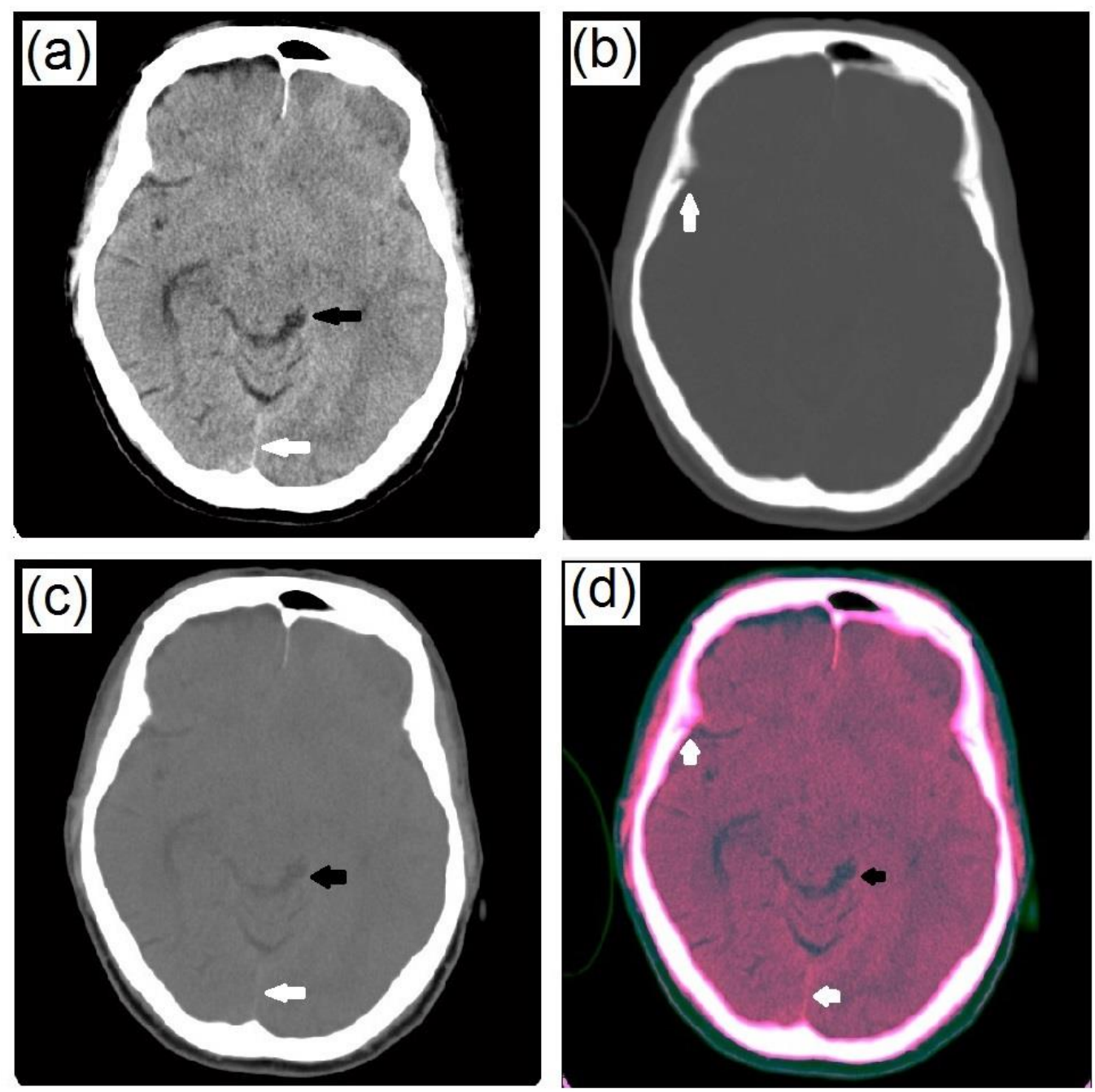

Implementation the multiplewindows blending to the head image of patient is shown in Figure 9, using a combination of different window settings. Again, the blended images have excellent contrast. For example, in the blending of the brain-bone-soft tissue windows, the temporal bone (indicated by the white vertical arrow), the quadrigeminal cistern (indicated by the black horizontal arrow), and the falx cerebri (indicated by the white horizontal arrow) all have very high contrast. The details of the temporal bone usually appear only in the bone window setting, and the quadrigeminal cistern and falx cerebri only usually appear clearly in the brain window setting.

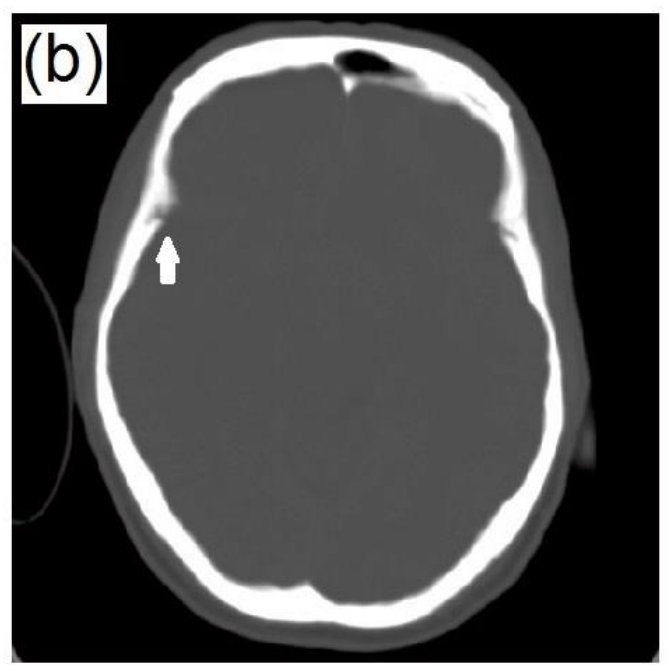

Figure 9. Image of multiple-windows blending of brain. (a) Brain window, (b) Bone window, (c) Soft tissue window, and (d) Brain-bone-soft tissue windows. 
Implementation the multiplewindows blending to the thorax is shown in Figure 10. The blended images have excellent contrast (d). For example, in the blending of the soft tissue-bone-lung windows, the soft tissue, bone and lung all have very high contrast. Some parts look very clear, such as the descending aorta (indicated by the white horizontal arrow), the ribs (indicated by black horizontal arrow), and the pulmonary vessels (indicated by white vertical arrow). The results are significantly different from the images visualized using one window, in which the descending aorta is only seen in the soft tissue window (a),
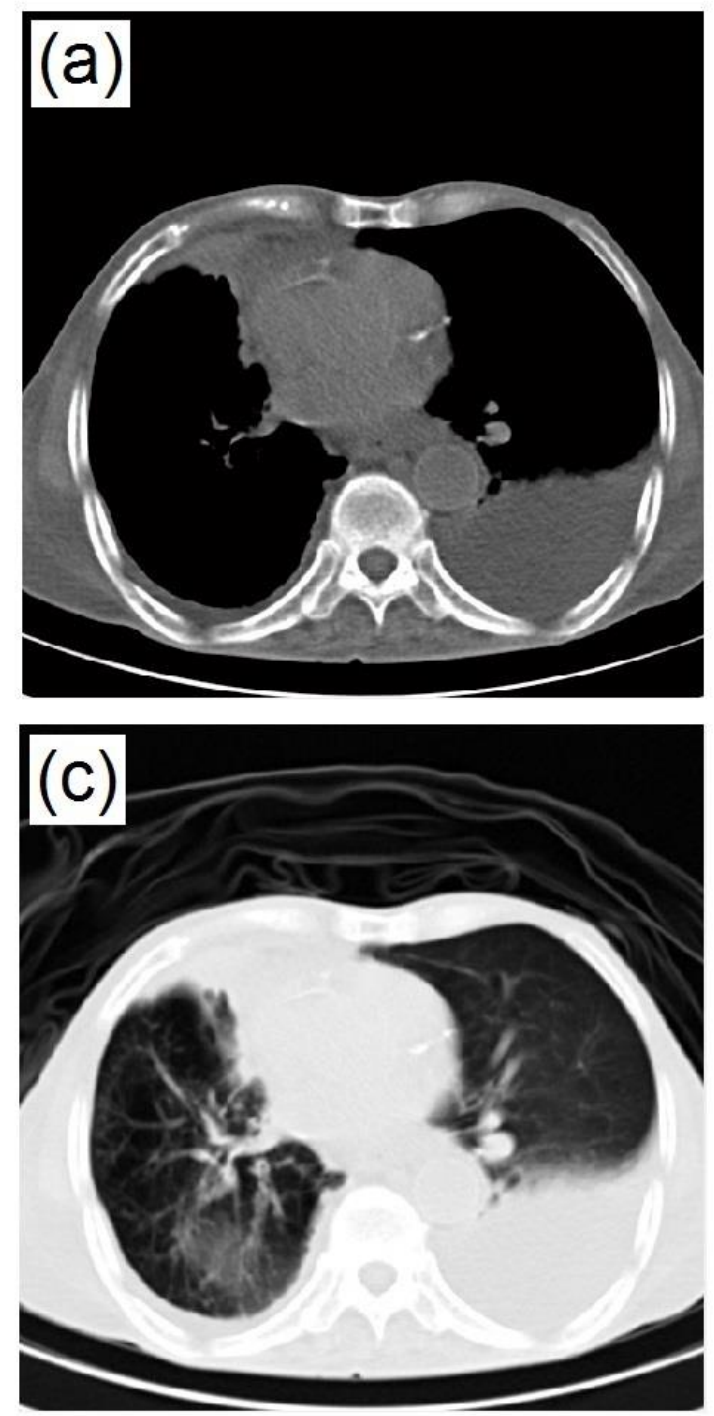

the rib detail is only visible in the bone window (b), and the pulmonary vessel is visible only in the lung window (c).

\section{Discussion}

In this paper, we have proposed a new method of CT scan image representation using multiple-windows blending in RGB color space. The proposed method has been implemented in the TOS-phantom with various objects, the thoracic anthropomorphic phantom, head of patient, and thorax of patient.
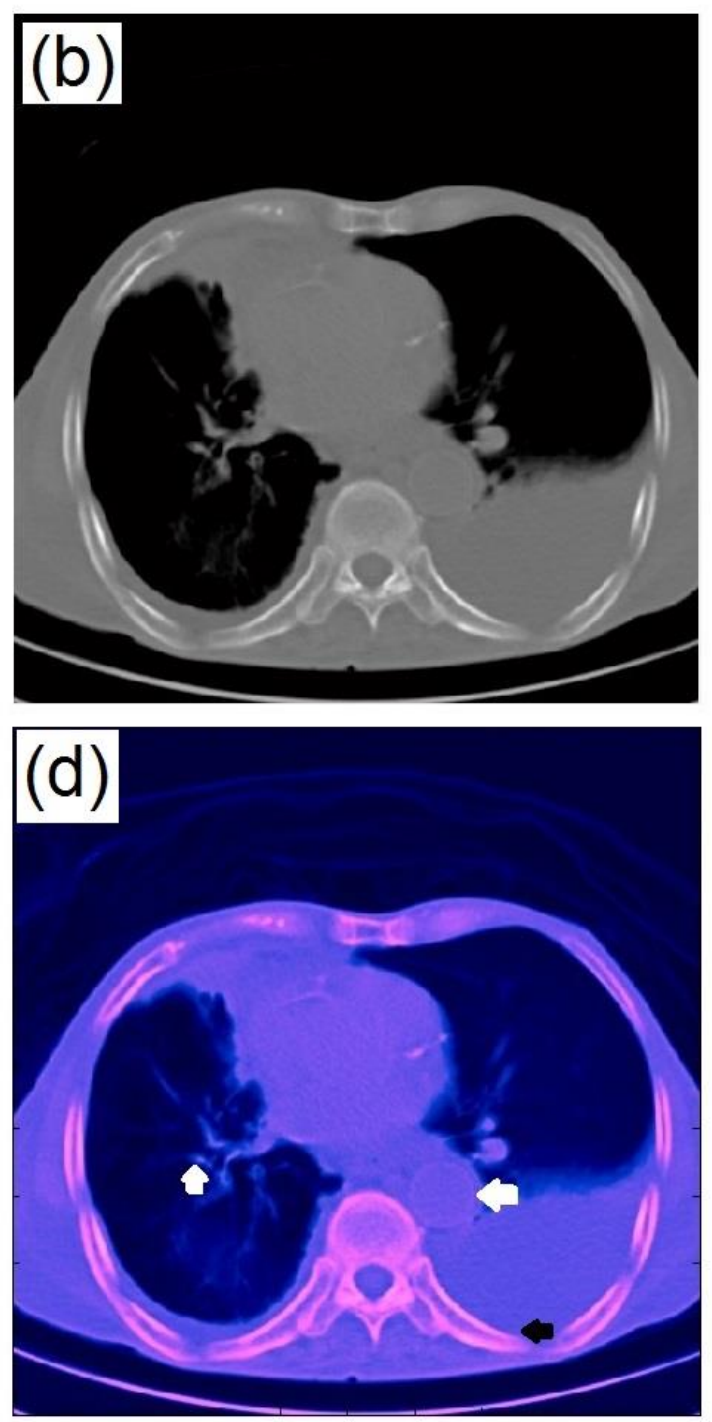

Figure 10. Image of multiple-windows blending of thorax. (a) Soft tissue window, (b) Bone window, (c) Lung window, and (d) Soft tissue-bone-lung windows. 
The method enables the result of multiple windows to be presented in a single view, so that users do not need to make any changes to the windows settings. This may fasten the interpretation time because more information is viewed in a single image. Previously, AHE, CLAHE, MAHE and RADIO algorithms allowed shorter interpretation time than conventional windows $[19,20,22,26]$. This novel multiplewindows blending is able to generate images with high contrast for many tissues of interest simultaneously, according to the initial settings of the specified windows. For instance, in TOS-phantom image, the liver, soft tissue, and lung windows produces an image in which the objects of air, Delrin $($, acrylic, nylon, and polypropylene can be clearly differentiated, and in the thoracic image of anthropomorphic phantom, the blending of soft tissue, bone and lung windows produces an image in which the details of soft tissues, bones, and lung nodules can be seen simultaneously. These representations cannot be accomplished using conventional window techniques.

This novel method is different from the previous methods of AHE [18, 19], CLAHE [20], and MAHE [21, 22], and the previous multi-windows blending method proposed by Mandell et al $[23,26]$, which combines multiple windows into a single grayscale image using their RADIO algorithm. In these previous methods, the pixel values representation may be inconsistent and can cause misinterpretation among practitioners. For example, the pulmonary vessel and bone may be displayed as the same gray level. However, this is not the case with our proposed method because, although the pulmonary vessel and bone appear at once in a single image, they appear with different colors. This minimizes any possible misinterpretation.

It is important to note that multiplewindows blending in RGB space is a novel concept and there is no standardization yet. For example, in this study, the thoracic image of the anthropomorphic phantom used the soft tissue, bone and lungs windows. The combination of three windows need to be optimized by expert radiolo- gists. This optimization requires further comprehensive study. After the optimization of the color arrangements, standardization is also required. For example, thoracic images may be standardized using three windows: the soft tissue window as the red plane, the lung window as the green plane, and the bone window as the blue plane. This standardization should reduce any uncertainty in interpreting multiplewindows blended images.

Since radiologists have only been trained to interpret single-window grayscale images, they will face some difficulties in interpreting multiple-window RGB images. However, the intuitive appearance and the potential benefits offered by this multiple-windows blending may persuade them to adapt to such images in the near future. Since our method uses RGB color images, which is new in CT scans and it is only implemented in the phantoms, further studies are required to evaluate the results in the case of a patient with a particular abnormality.

\section{Conclusions}

Multiple-windows blending to display CT images in a single RGB color image has been successfully developed, and implemented in phantoms and patients images. It is able to visualize with high contrast many tissues of interest for very different groups of densities in a single view simultaneously. However, this novel method requires optimization and standardization, and needs an adaptation in the skills of the radiologist. Further studies are needed to explore clinical applications of this novel image representation.

\section{Acknowledgments}

This work was funded by the Penelitian Sumber Dana Selain APBN Fakultas Sains \& Matematika UNDIP Tahun Anggaran 2019, contract number. 4936/UN7.5.8/PP/2019. 


\section{References}

1. Verdun F.R., Meuli R.A., Bucher G., Noel A., Stines J., Schnyder P., Valley J.F. Dose and image quality characterisation of CT units. Radiation Protection Dosimetry, Vol. 90, Nos 1-2, 2000, pp. 193-196.

2. Anam C., Haryanto F., Widita R., Arif I. New noise reduction method for reducing CT scan dose: Combining wiener filtering and edge detection algorithm. AIP Conference Proceedings, Vol. 1677, No 1, 2015, pp. 040004.

3. Anam C., Fujibuchi T., Budi W.S. Haryanto F., Dougherty F. An algorithm for automated modulation transfer function measurement using an edge of a PMMA phantom: Impact of field of view on spatial resolution of CT images. Journal of Applied Clinical Medical Physics, Vol. 19, No 6, 2018, pp. 244252.

4. Fukuda A., Lin P.P., Matsubara K., Miyati T. Measurement of gantry rotation time in modern CT. Journal of Applied Clinical Medical Physics. Vol. 15, No 1, 2014, pp. 303-308.

5. McCollough C.H., Yu L., Kofler J.M., Leng S., Zhang Y., Li Z., Carter R.E. Degradation of CT low-contrast spatial resolution due to the use of iterative reconstruction and reduced dose levels. Radiology, Vol. 276, No 2, 2015, pp. 499-506.

6. Gulliksrud K., Stokke C., Martinsen A.C.T. How to measure CT image quality: Variations in CT-numbers, uniformity and low contrast resolution for a CT quality assurance phantom. Physica Medica, Vol. 30, No 4, 2014, pp. 521-526.

7. Kalender W.A. X-ray computed tomography. Physics in Medicine and Biology, Vol. 51, pp. R29-R43.

8. Babbel R., Harnsberger H.R., Nelson B., Sonkens J., Hunt S. Optimization of techniques in screening CT of the sinuses. American Journal of Neuroradiology, Vol. 12, 1991, pp. 849-854.

9. Anam C., Haryanto F., Widita R., Arif I., Dougherty G. Automated calculation of water equivalent diameter (Dw) based on AAPM task group 220. Journal of Applied Clinical Medical Physics, Vol 17, No 1, 2016, pp. 320-333.

10. Bach A.M., Panicek D.M., Schwartz L.H., Herman S.K., Ho M.N., Castellino R.A. CT bone window photography in patients with cancer. Radiology, Vol. 197, 1995, pp. 849-852.

11. Costelloe C.M., Chuang H.H., Chasen B.A., Pan T., Fox P.S., Bassett R.L., Madewell J.E. Bone windows for distinguishing malignant from benign primary bone tumors on FDG PET/CT. Journal of Cancer, Vol. 4, No 7, 2013, pp. 524-530.

12. Pomerantz S.M., White C.S., Krebs T.L., Daly B., Sukumar S.A., Hooper F., Siegel E.L. Soft tissue and bone window settings for soft-copy interpretation of chest and abdominal CT. American Journal of Roentgenology, Vol. 174, 2000, pp. 311-314.

13. Xue Z., Antani S., Long L.R., DemnerFushman D., Thoma G.R. Window classification of brain CT images in biomedical articles. AMIA Annu Symp Proc, Vol. 2012, 2012, pp. 1023-1029.

14. Mayo-Smith W.W., Gupta H., Ridlen M.S., Brody J.M., Clements N.C., Cronan J.J. Detecting hepatic lesions: The added utility of CT soft tissue window settings. Radiology, Vol. 210, 1999, pp. 601-604.

15. Sabouri S., Khatami A., Azadeh P., Ghoroubi J., Azimi G.H. Adding soft tissue window setting to the standard abdominal CT scan protocol: Is it useful? Iranian Journal of Radiology, Vol. 5, No 2, 2008, pp. 65-70.

16. Takagi S., Nagase H., Hayashi T., Kita T., Hayashi K., Sanada S., Koike M. Combined multi-kernel head computed tomography images optimized for depicting both brain parenchyma and bone. J of X-Ray Science and Technology, Vol. 22, 2014, pp. 369-376.

17. Takagi S., Nagase H., Hayashi T., Kita T., Hayashi K., Sanada S., Koike M. Combined multi-kernel chest computed tomography images optimized for de- 
picting both lung and soft tissue. Clinical Imaging, Vol. 38, 2014, pp. 104108.

18. Pizer S.M., Zimmerman J.B., Staab E.V. Adaptive grey level assignment in CT scan display. Journal of Computer Assisted Tomography, Vol. 8, 1984, pp. 300-308.

19. Lehr J.L., Capek P. Histogram equalization of CT images. Radiology, Vol. 154, 1985, pp. 163-169.

20.Pizer S.M., Amburn E.P., Austin J.D., Cromartie R., Greer T., Romeny B.T.H., Zimmerman J.B., Zuiderveld K. Adaptive histogram equalization and its variations. Computer Vision, Graphics and Image Processing, Vol. 39, 1987, pp. $355-368$.

21. Jin Y., Fayad L.M., Laine A.F. Contrast enhancement by multi-scale adaptive histogram equalization. In: Aldroubi A, Laine AF, Unser MA, eds. Wavelet applications and signal and image processing IX. Proceedings of SPIE 2001.

22. Fayad L.M., Jin Y., Laine A.F., Berkmen Y.M., Pearson G.D., Freedman B., Heertum R.V. Chest CT window settings with multiscale adaptive histogram equalization: Pilot study. Radiology, Vol. 223, 2002, pp. 845-852.

23. Mandell J.C., Khurana B., Folio L.R., Hyun H., Smith S.E., Dunne R.M., Andriole K.P. Clinical applications of a CT window blending algorithm: RADIO (relative attenuation-dependent image overlay). Journal of Digital Imaging, Vol. 30, 2017, pp. 358-368.

24. Kim Y.J., Lee S.H., Kim T.Y., Park J.Y., Choi S.H., Kim K.G. Body fat assessment method using CT images with separation mask algorithm. Journal of Digital Imaging, Vol. 26, 2013, pp. 155162.

25. Yoshizumi T., Nakamura T., Yamane M., Islam A.H.M.W., Menju M., Yamasaki K., Arai T., Kotani K., Funahashi T., Yamashita S., Matsuzawa Y. Abdominal fat: Standardized technique for measurement at CT. Radiology, Vol. 211, 1999, pp. 283-286.
26. Mandell J.C., Wortman J.R., Rocha T.C., Folio L.R., Andriole K.P., Khurana B. Computed tomography window blending: Feasibility in thoracic trauma. Academic Radiology, Vol. 29, No 9, 2018, pp. 1190-1200. 